\title{
Esami diagnostici nel sospetto di carcinoma midollare della tiroide
}

\author{
Antonio Matrone ${ }^{1} \cdot$ Carla Gambale $^{1} \cdot$ Rossella Elisei $^{1}$
}

Accettato: 30 agosto 2021 / Pubblicato online: 29 ottobre 2021

(c) The Author(s) 2021

\section{Introduzione}

Il carcinoma midollare della tiroide (CMT) è una neoplasia rara che rappresenta il 5-10\% dei carcinomi tiroidei e può presentarsi in forma sporadica $(75 \%)$ o in forma ereditaria (25\%). Il decorso clinico e l'esito finale del CMT sono piuttosto eterogenei e condizionati da diversi fattori. La presenza di metastasi linfonodali e/o a distanza al momento della diagnosi influenza negativamente la prognosi [1]. Al momento della diagnosi, il coinvolgimento linfonodale è piuttosto frequente e le metastasi a distanza sono presenti in circa il $10 \%$ dei pazienti. L'importanza della diagnosi precoce risiede nella possibilità di trattare precocemente la malattia migliorandone l'andamento clinico in termini di recidiva e di sopravvivenza.

\section{Work-up diagnostico del CMT}

Attualmente, diversi strumenti vengono utilizzati per il work-up diagnostico del CMT, alcuni dei quali con bassa sensibilità (ecografia ed esame citologico su agoaspirato, FNAC), altri con elevata sensibilità ma a scapito della specificità, soprattutto per valori borderline (calcitonina, CT). Inoltre, la presenza e il tipo della mutazione germinale del gene $R E T$ risulta fondamentale per la diagnosi nelle forme ereditarie.

\section{Ecografia tiroidea}

A differenza del carcinoma differenziato della tiroide (CDT), il ruolo diagnostico dell' ecografia nel CMT è molto

\footnotetext{
$\triangle$ R. Elisei

rossella.elisei@med.unipi.it

1 U.O. Endocrinologia 1, Dipartimento di Medicina Clinica e Sperimentale, Università di Pisa, Pisa, Italia
}

dibattuto. Studi recenti mostrano come non ci siano caratteristiche ecografiche patognomoniche di CMT [2], e i noduli risultati CMT all'esame istologico possono presentare un aspetto ecografico non sospetto per malignità. Inoltre, non sono disponibili classificazioni ecografiche di riferimento per il CMT, e quelle standardizzate ed efficaci per il carcinoma papillare della tiroide sembrano non essere altrettanto valide per il CMT [2].

\section{Esame citologico su agoaspirato}

Il FNAC, che rappresenta il gold standard diagnostico per il CDT, mostra scarsa sensibilità per individuare il CMT. Infatti, un'alta percentuale di noduli risultati CMT all'istologia non presenta un risultato citologico indicativo o suggestivo per CMT [3]. Proprio per questo motivo, inoltre, sulla base delle caratteristiche ecografiche del nodulo, non sussisterebbe neanche indicazione all'esecuzione di FNAC [3].

Il dosaggio della CT su liquido di lavaggio [4, 5] e l'immunocitochimica rappresentano test addizionali che migliorano la sensibilità di FNAC ma, non essendo procedure di routine, per poter essere eseguite occorre quanto meno un sospetto di CMT come, ad esempio, quello derivante da un valore elevato di CT sierica.

\section{Dosaggio della calcitonina sierica}

\section{Calcitonina basale}

Il dosaggio della CT sierica, nei pazienti con patologia nodulare tiroidea, rappresenta lo strumento di screening più sensibile e specifico per il CMT [6].

Pazienti con CMT diagnosticato mediante riscontro di valori elevati di CT in corso di valutazione di patologia no- 
dulare tiroidea presentano diagnosi precoci che permettono un trattamento chirurgico di tumori meno avanzati con prognosi migliore [7].

Nonostante queste evidenze, il dosaggio routinario della CT nel work-up diagnostico del nodulo tiroideo non è raccomandato da tutte le società scientifiche. I limiti della procedura sono rappresentati da una mancata interpretazione univoca dei risultati, dovuta alla presenza di diversi sistemi di dosaggio, ai possibili falsi positivi da potenziali condizioni interferenti (es. anticorpi eterofili, terapia con inibitori di pompa protonica, produzione ectopica da altri tumori avanzati), alla presenza di altre patologie tiroidee e non (es. tiroidite cronica autoimmune, insufficienza renale, iperparatiroidismo, altri tumori neuroendocrini), alle differenze legate all'età (valori di CT più elevati nei primi 3 anni di età) e al sesso (valori di CT più elevati in età adulta nell'uomo rispetto alla donna) e, infine, al rapporto costo-beneficio.

Valori di calcitonina sierica $\geq 100 \mathrm{pg} / \mathrm{mL}$, sono fortemente suggestivi di CMT [8]. Lavori recenti hanno cercato di determinare dei cut-off di CT sierica diagnostici per il CMT, differenti in base al sesso. Per i maschi un valore di CT basale $>34 \mathrm{pg} / \mathrm{ml}$, ha mostrato una sensibilità dell' $88,9 \%$ e una specificità del $95 \%$ nell'identificare il CMT; tale valore per le femmine era $>30$ pg/ml (sensibilità $75,9 \%$, specificità $93,7 \%$ ) [9].

Inoltre, i valori di CT sierica sono direttamente proporzionali alle dimensioni della neoplasia e al grado di estensione della malattia: più la malattia è estesa e presenta metastasi linfonodali e/o a distanza, più i valori di CT sono elevati [10].

Nel caso di valori di CT superiori al range di normalità ma non chiaramente suggestivi per CMT, è opportuno eseguire un test di stimolo, eseguire un dosaggio della CT nel liquido di lavaggio del FNAC [4, 5] e chiedere al patologo una immunocitochimica per CT.

\section{Calcitonina stimolata}

Il test di stimolo per CT si esegue oggi utilizzando il calcio gluconato. Tale test consente di: 1) escludere i falsi positivi che presentano una risposta allo stimolo minimale o assente (test piatto); 2) confermare il CMT nei casi in cui la CT dopo stimolo sia aumentata al di sopra dei valori considerati diagnostici. Il test si esegue con un'infusione di $2-2,5 \mathrm{mg} / \mathrm{kg}$ di peso corporeo di calcio elemento diluiti fino a $50 \mathrm{ml}$ con soluzione fisiologica $(\mathrm{NaCl} 0,9 \%)$ da infondere in 5-10 minuti per via endovenosa. La CT viene generalmente dosata prima dell'infusione (tempo 0) e dopo 2, 5 e 10 minuti dal termine della stessa. Il calcolo della quantità di calcio gluconato da infondere dovrebbe essere eseguito, per evitare il sovradosaggio in particolar modo nei soggetti obesi, tendendo conto del peso ideale, piuttosto che del peso reale (http:// www.manuelsweb.com/IBW.htm).
I risultati del test vanno interpretati caso per caso, tenendo in considerazione i valori di CT suggeriti da alcuni recenti lavori che mostrano come valori stimolati $>466 \mathrm{pg} / \mathrm{ml}$ (sensibilità 94,4\%, specificità 80\%) nei maschi e $>79$ pg/ml (sensibilità: $100 \%$, specificità: $50 \%$ ) nelle femmine, presentino miglior specificità e sensibilità per la diagnosi di CMT [9].

\section{Analisi genetica}

Tutti i casi di CMT, sia quelli che si presentano all'esordio come ereditari sia quelli che si presentano come sporadici, devono essere sottoposti allo screening genetico di RET. RET è l'oncogene responsabile delle forme ereditarie. Lo screening si esegue mediante analisi del DNA costituzionale estratto o dal sangue o da altri tessuti biologici. La mutazione germinale è trasmessa con carattere autosomico dominante ed è diagnostica di ereditarietà. La correlazione genotipo-fenotipo consente anche di prevedere se si tratterà di una forma MEN 2A, 2B o FMTC [11].

\section{Conclusioni}

La diagnosi pre-chirurgica di CMT non è sempre facile. L'ecografia e la citologia hanno una scarsa sensibilità e non sono capaci di identificare circa il 50\% dei casi. La CT sierica è molto sensibile ma, per valori positivi $<100 \mathrm{pg} / \mathrm{ml}$, può perdere in specificità. In questi casi, la CT non deve essere considerata diagnostica di CMT ma piuttosto deve rappresentare un indicatore a eseguire ulteriori indagini, quali il dosaggio della $\mathrm{CT}$ nel liquido di lavaggio dell'ago usato per eseguire FNAC, l'immunocitochimica per CT sul materiale citologico agoaspirato e il test di stimolazione con calcio per CT. L'integrazione dei risultati di questi test può aumentare significativamente la capacità diagnostica di CMT. Una volta posta la diagnosi di CMT, lo screening genetico di RET è fondamentale per identificare le forme ereditarie.

Funding Note Open access funding provided by Università di Pisa within the CRUI-CARE Agreement.

Conflitto di interesse Gli autori Antonio Matrone, Carla Gambale e Rossella Elisei dichiarano di non avere conflitti di interesse.

Consenso informato Lo studio presentato in questo articolo non ha richiesto sperimentazione umana.

Studi sugli animali Gli autori di questo articolo non hanno eseguito studi sugli animali.

Nota della casa editrice Springer Nature rimane neutrale in riguardo alle rivendicazioni giurisdizionali nelle mappe pubblicate e nelle affiliazioni istituzionali. 
Open Access This article is licensed under a Creative Commons Attribution 4.0 International License, which permits use, sharing, adaptation, distribution and reproduction in any medium or format, as long as you give appropriate credit to the original author(s) and the source, provide a link to the Creative Commons licence, and indicate if changes were made. The images or other third party material in this article are included in the article's Creative Commons licence, unless indicated otherwise in a credit line to the material. If material is not included in the article's Creative Commons licence and your intended use is not permitted by statutory regulation or exceeds the permitted use, you will need to obtain permission directly from the copyright holder. To view a copy of this licence, visit http://creativecommons.org/licenses/by/4.0/.

\section{Bibliografia}

1. Kotwal A, Erickson D, Geske JR et al (2021) Predicting outcomes in sporadic and hereditary medullary thyroid carcinoma over two decades. Thyroid 31(4):616-626

2. Matrone A, Gambale C, Biagini M et al (2021) Ultrasound features and risk stratification systems to identify medullary thyroid carcinoma. Eur J Endocrinol 185(2):193-200

3. Workman AD, Soylu S, Kamani D et al (2021) Limitations of preoperative cytology for medullary thyroid cancer: proposal for improved preoperative diagnosis for optimal initial medullary thyroid carcinoma specific surgery. Head Neck 43(3):920-927

4. Boi F, Maurelli I, Pinna G et al (2007) Calcitonin measurement in wash-out fluid from fine needle aspiration of neck masses in patients with primary and metastatic medullary thyroid carcinoma. J Clin Endocrinol Metab 92(6):2115-2118

5. Brigante G, Madeo B, Diazzi C et al (2021) Ruolo diagnostico del dosaggio della calcitonina su liquido di lavaggio da agoaspirato tiroideo nel carcinoma midollare della tiroide. Endocrinologo 22:93-97

6. Elisei R (2008) Routine serum calcitonin measurement in the evaluation of thyroid nodules. Best Pract Res Clin Endocrinol Metab 22(6):941-953

7. Elisei R, Bottici V, Luchetti F et al (2004) Impact of routine measurement of serum calcitonin on the diagnosis and outcome of medullary thyroid cancer: experience in 10,864 patients with nodular thyroid disorders. J Clin Endocrinol Metab 89(1):163-168

8. Costante G, Meringolo D, Durante C et al (2007) Predictive value of serum calcitonin levels for preoperative diagnosis of medullary thyroid carcinoma in a cohort of 5817 consecutive patients with thyroid nodules. J Clin Endocrinol Metab 92(2):450-455

9. Fugazzola L, Di Stefano M, Censi S et al (2021) Basal and stimulated calcitonin for the diagnosis of medullary thyroid cancer: updated thresholds and safety assessment. J Endocrinol Invest 44(3):587-597

10. Machens A, Dralle H (2010) Biomarker-based risk stratification for previously untreated medullary thyroid cancer. J Clin Endocrinol Metab 95(6):2655-2663

11. Elisei R, Alevizaki M, Conte-Devolx B et al (2013) 2012 European thyroid association guidelines for genetic testing and its clinical consequences in medullary thyroid cancer. Eur Thyroid $\mathbf{J}$ 1(4):216-231 